\title{
Online Resource and ICT-Aided Virtual Laboratory Setup
}

\author{
Antara Mahanta \\ Department of Electronics and Communication \\ Engineering Gauhati University, \\ Guwahati-781014, Assam, India
}

\author{
Kandarpa Kumar Sarma \\ Department of Electronics and Communication \\ Engineering Gauhati University, \\ Guwahati-781014, Assam, India
}

\begin{abstract}
The extensive use of Information and Communication Technology (ICT) has enabled the development of new ways of teaching and learning. ICT provides flexibility to a learner which is denied by the traditional learning process. This paper proposes an integrated structure of virtual laboratory setup to conduct online laboratory sessions for UG/PG level courses of engineering programmes. The setup provides an access based request of multiple users from remote locations to conduct the experiments online. The web based facility enables a student to repeat the process multiple times to learn theoretical as well as practical aspects of their subjects. In our approach, we have used LabVIEW 2011 to develop the interface panels for remote triggering operation from client side. With this approach students are provided the facilities to perform experiments from remote locations. The access to the facility is through an authentication mechanism through which the students are allowed to use the resources provided in the repository. The advantage of the system is that it is re-usable and the students can repeat the experiments a number of times till their access session time is over.
\end{abstract}

\section{Keywords}

ICT, Virtual Laboratory, LabVIEW, Web Resources, Experiments

\section{INTRODUCTION}

The prevalence of Information and Communication Technology (ICT) has been extensively increases in the education field by developing new ways of teaching and learning process. ICT provides flexibility to a learner which is denied by the traditional learning process. Web based learning has become an important part of education which improve the long distance learning and remote laboratories. The use of internet facilitates the student to conduct their experiments without physically being in the laboratory by using remotely triggered virtual laboratory setup [1]. This would save a lot of resources like setting up a whole lab in many locations. The designed website supports for conducting a few selected laboratory experiments of Basic Electronics course. The student can access the virtual laboratory and perform experiments without any limitations of time and location. They can observe the outputs of experiments by changing the parameters and evaluating the results. The design of this virtual laboratory involves the use of web browser and an application software package of National Instrument called LabVIEW (Laboratory Virtual Instrumentation Engineering Workbench) $[2,3]$.

LabVIEW is a power and flexible instrumentation and analysis software system which provide an active and controllable interface for test, measurement, instrument control, data processing and data analysis applications. The program developed in LabVIEW is known as Virtual Instrument (VI) which contains three main components: the front panel, the block diagram, and icon of connector pane. The user interacts with program through the front panel which includes display, control and indicator for input and output terminals. Graphical source code called a block diagram and the connector pane defines the inputs and outputs of the experiments. In our approach, we have used LabVIEW 2011 to develop the interface panels for remote triggering operation from client side. With this approach students are provided the facilities to perform experiments from remote locations. The access to the facility is through an authentication mechanism through which the students are allowed to use the resources provided in the repository. The remote triggered virtual labs thus evolved as the powerful tool in this regard of experimentation.

\section{ICT ASSISTED VLAB}

ICT plays a vital role in the design and implementation of virtual laboratory. Virtual laboratory is an excellent tool in both conventional and distance learning to emphasize science and technology education by providing flexibility to students and teachers. The development of web based VLAB is divided into two types. They are real time implementation laboratory and remote simulation laboratory [4]. Real time implementation laboratory needs real devices for the experiments but the learners are at distant from the real laboratory. It allows the users to conduct the experiment in the real lab by controlling certain parameters. Remote simulation laboratory is the simulation program of real experiment on computer. Computer utilities are used to simulate complicated, expensive devices or to replace environmentally hazardous laboratory experiments. But simulators do not support the real instrument control via internet. Both the types of laboratory provides access to perform experiments from anywhere through web browser environment [5].

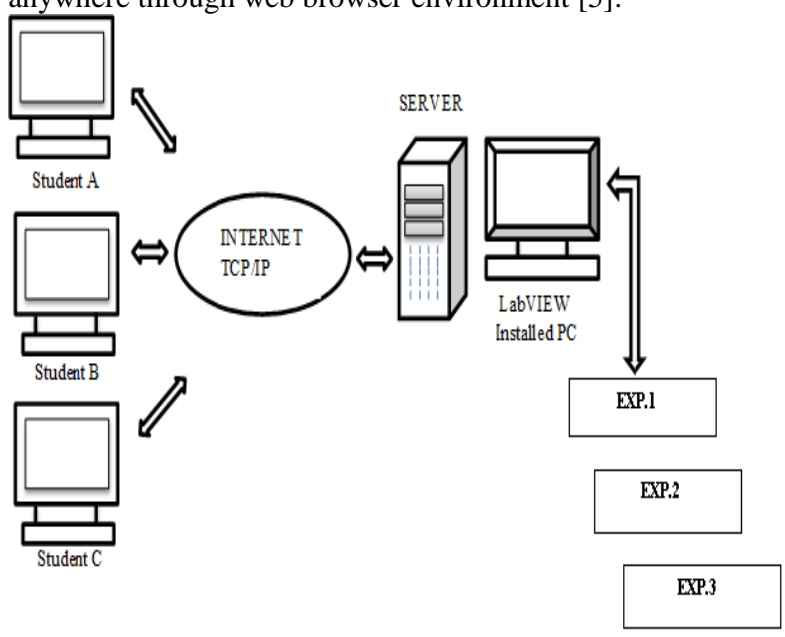

Fig. 1 General Overview of Remotely Accessible Laboratory 


\subsection{System Structure}

The virtual laboratory system involves client server architecture [6]. The structure of client-server based virtual laboratory is shown in Figure 1. The user interfaces for all the experiments have been designed in a way to give the remote client a basic understanding and insight of the principles involved. The client sends a request to the remote server which includes LabVIEW 2011software package. The request is processed and replies with a response which is then interpreted and displayed by the client application. The user's computer must have LabVIEW run-time engine installed to access the remote front panels. LabVIEW remote front panels allow users to view and control VIs through a standard Web browser. A remote front panel web page can be created with the LabVIEW Web Publishing Tool [7,8]. The published VI must be running in the server to observe and control from remote locations. Once the server closes the VI experiments it cannot be carried out remotely.

\subsection{Existing Performance of Vlab}

Remote triggered virtual labs can significantly reduce the rate of initial purchase of equipment by simulating high cost experiments. Vlab can also eliminate the risk related with running of high powered equipment in traditional labs. It also acts as an asynchronous tool for the users which can access from anywhere and anytime. However, a major disadvantage is that if any network problem arises or the user may make any mistakes during the process then the system will be affected and terminate the execution of the application. Table 1 summarizes the performance of virtual laboratory to conduct the practical sessions from any distant place.

Table 1. Performance of the Vlab Setup

\begin{tabular}{|l|l|}
\hline \multicolumn{1}{|c|}{ Advantages } & \multicolumn{1}{c|}{ Drawbacks } \\
\hline & \multicolumn{1}{c|}{ May get virus effected } \\
Cost effectiveness & Network Problems \\
Safety & Slowdown in simultaneous \\
Flexibility & access of multiple users \\
Feasibility & \\
\hline
\end{tabular}

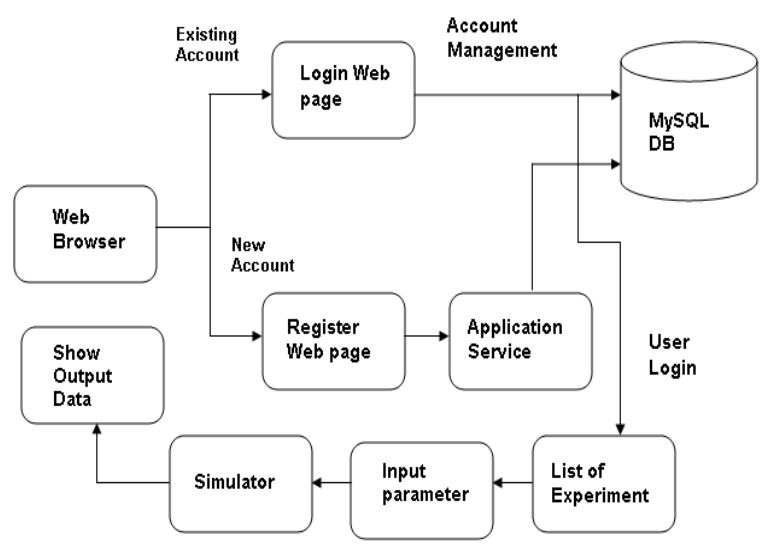

Fig.2 Vlab Web Portal Architecture

\section{ONLINE WEB RESOURCES}

In this section, we describe the resources to support the virtual laboratory session. The experimental setups connected to the server can be accessed through a web portal which can be controlled using a login/password authentication [9]. The Vlab web portal components are being built for handling user accounts, remote observation/operation, experiment evaluation and data management. The Vlab web portal architecture is shown in Figure 2. The web portal interface comprises of: (a) web page that allow login for registered user to get access to the system and (b) web page that enable to register the user for application services. All the registered information entered by the user is stored in the data base which can be retrieving for future use. Once the user successfully logins, he/she can view the various options available for him/her to work on virtual labs. A user can do an experiment by selecting it from the List of Experiment page. The detail of the experiment like theory, procedure, quiz, reference etc. can also observe by selecting the respective web pages. Now, the user can select the various parameters to obtain the desired results by using simulator page. The administrator or the teacher has the privileges to create new VIs in the web portal and to update the progress of the experiment for the remote users to monitor the experiment progress [10-12]. The resources used for the framework are shown in Table 2.

Table 2. Resources used for development of Vlab setup

\begin{tabular}{|c|c|c|}
\hline $\begin{array}{c}\text { Serial } \\
\text { No. }\end{array}$ & Item & Description \\
\hline 1 & Server & $\begin{array}{l}\text { HP ProLiant DL580G5 } \\
\text { Quad-Core Intel Xeon } \\
\text { processors 4 GB RAM } \\
500 \text { GB HDD }\end{array}$ \\
\hline 2 & Operating System & Windows server 2003 \\
\hline 3 & Web Resource & IIS ASP/MS-SQL \\
\hline 4 & Internet Link & 1 Gbps lease line \\
\hline
\end{tabular}

\section{DESIGN OF EXPERIMENTS}

As part of the setup a few practical experiments are formulated. The access of these experimental setups is given as per the process mentioned in Section 3. The experiments provided are basic in nature which enables the students to repeat and perform them whenever they are free or willing.

Table 3: Selected List of Experiments Conduct Remotely

\begin{tabular}{|c|l|}
\hline Group of subjects & \multicolumn{1}{|c|}{ Experiments } \\
\hline Basic Electronics & $\begin{array}{l}\text { Verification of KVL and } \\
\text { KCL } \\
\text { Diode Characteristics } \\
\text { Rectifier circuits } \\
\text { Truth table verification of } \\
\text { gates }\end{array}$ \\
\hline Communication principle & $\begin{array}{l}\text { Amplitude Modulation } \\
\text { Frequency modulation }\end{array}$ \\
\hline
\end{tabular}




\subsection{Setup 1: Half Wave Rectification}

The experiment with input and output wave form is designed and published for remote experimentation. The user controls the amplitude of the input AC signal and it may vary from -20 $\mathrm{V}$ to $20 \mathrm{~V}$. Figure 3 and 4 shows the front panel and the block diagram of the VI program.

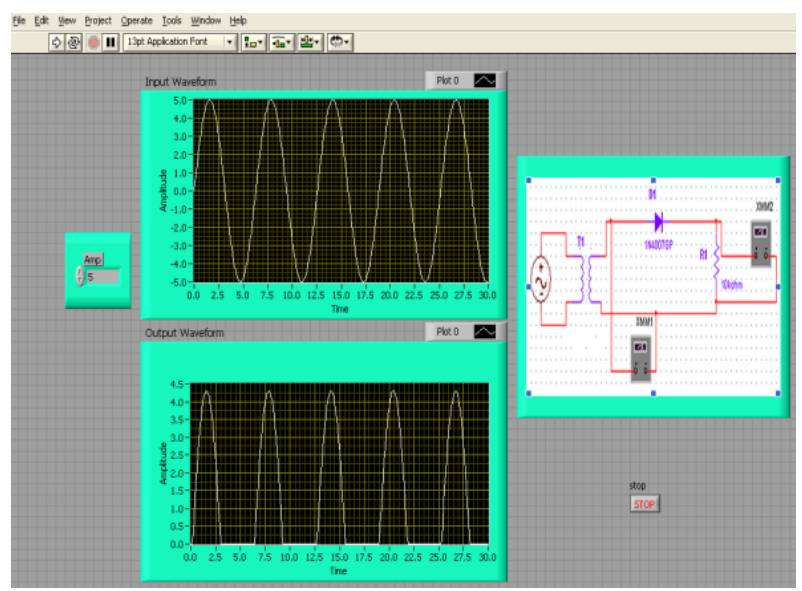

Fig. 3 Front panel of Half Wave Rectifier

\subsection{Setup 2: Amplitude Modulation}

Amplitude modulation is used for transmitting information via carrier wave. It works by varying the strength of the transmitted signal in relation to the information being sent. In this experiment, the modulated signal is generated by selecting the amplitude and frequency of the modulating and carrier signal. The LabVIEW running on the system communicates with the parameters entered by the users to generate and display the out signal on the client pc [13]. The experiment can be performed by slot booking system. After Login, the convenient time slot has to be booked by entering user name and password field to generate a key and an ID.

The front panel and the block diagram of the corresponding VI are shown in Figure 5 and 6 respectively.

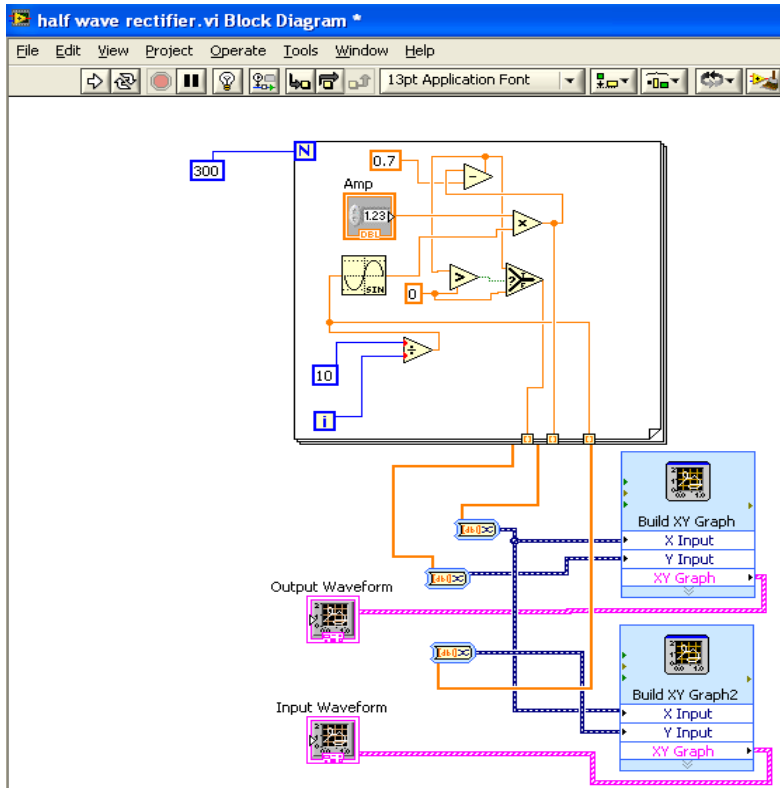

Fig. 4 Block Diagram of Half Wave Rectifier

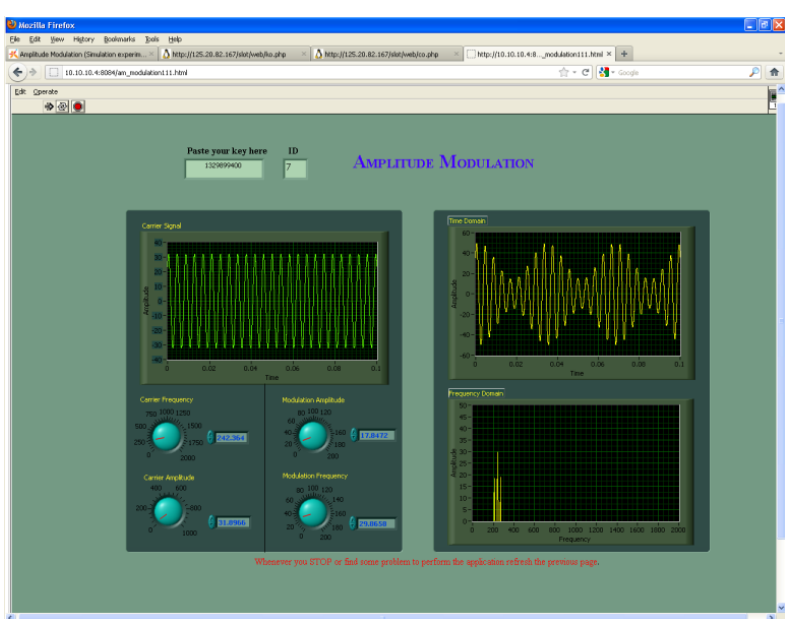

Fig. 5 Front Panel of Amplitude Modulation
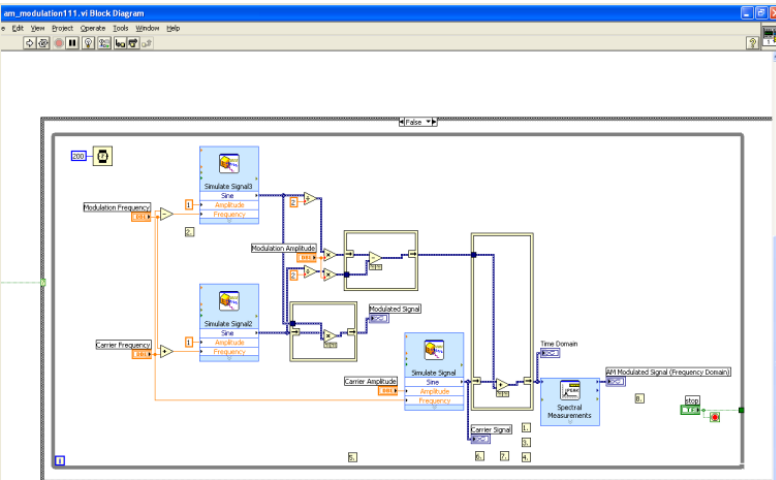

Fig. 6 Block Diagram of Amplitude Modulation

Table 3. Login Latency observed in Vlab experiments

\begin{tabular}{|c|c|c|}
\hline $\begin{array}{c}\text { Data rate } \\
\text { (kbps) }\end{array}$ & $\begin{array}{c}\text { No. of } \\
\text { users }\end{array}$ & $\begin{array}{c}\text { Access request } \\
\text { per second }\end{array}$ \\
\hline 52 & 5 & 0.05 \\
52 & 10 & 0.02 \\
52 & 15 & 0.018 \\
52 & 20 & 0.014 \\
\hline 126 & 5 & 0.14 \\
126 & 10 & 0.068 \\
126 & 15 & 0.046 \\
126 & 20 & 0.034 \\
\hline
\end{tabular}

\section{RESULT}

A summarized set of results derived from the number of user requests in different internet links are noted and presented in Table 3. It shows that the effectiveness of the system is dependent on the connectivity provided. As the number of users increases, the system slows down for the access of its clients. Further, if multiple users are expected to simultaneous connect to an instrument in real time; appropriate bandwidth has to be provisioned at the intermediate routers for the instrument traffic flow. The student first login by inserting the correct user name and password for authentication in the web 
page shown in figure 7. This is to make sure that only learners registered for the lab session are allowed to perform the remote experiments.

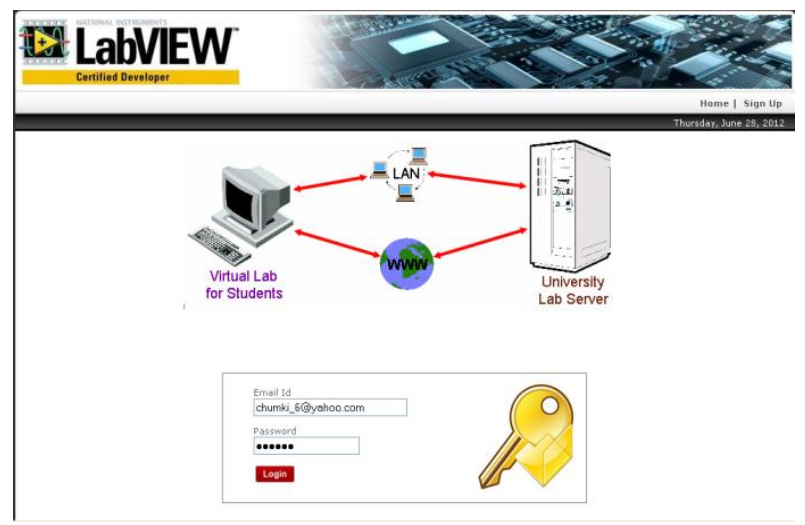

Fig.7 Login Interface of Vlab Web Portal

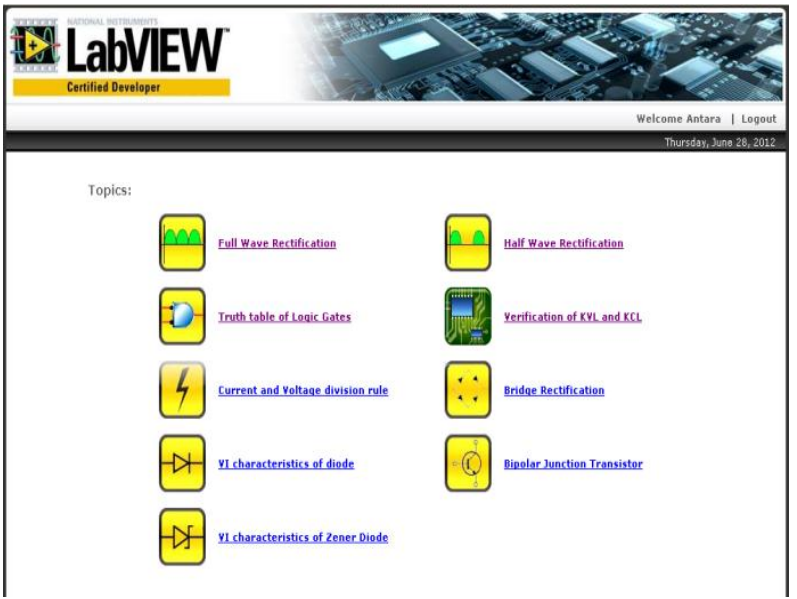

Fig. 8 Web Page of selecting Experiments

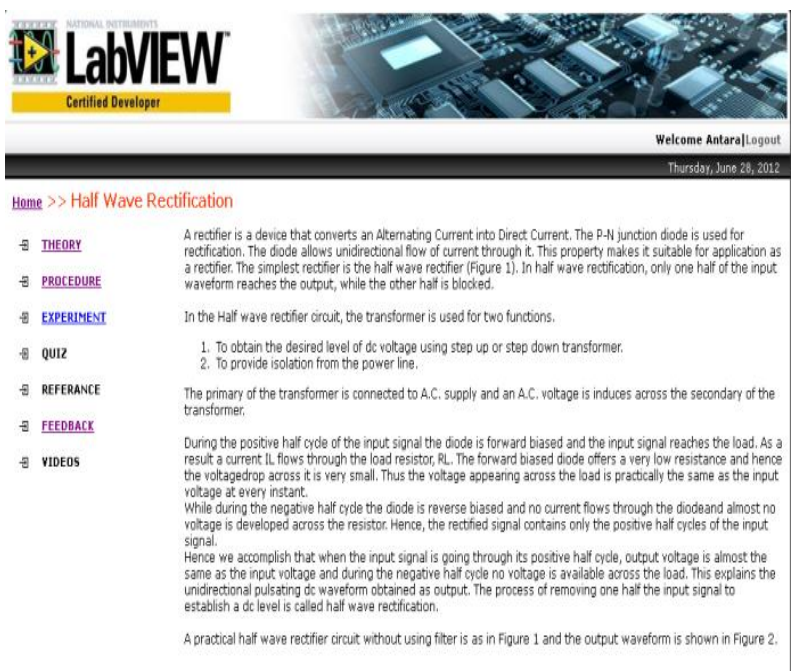

Fig. 9 Theory of HWR experiment

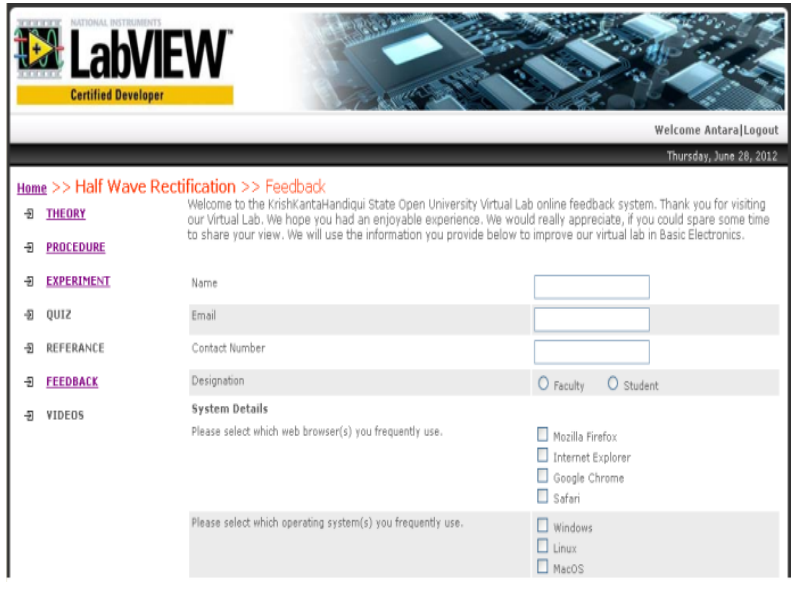

Fig. 10 Feedback Interface

After successful login the student shall now proceed to select the experiment from the list as shown in the web page of Figure 8. The user will now start conducting one experiment and continue with it by selecting various fields like theory, procedure, quiz, experiment, feedback etc. The theory interface of Half Wave Rectifier experiment and feedback interface as displayed on the screen of the client computer are shown in Fig.9 and Fig.10 respectively. Some of the earlier works were reported in $[14,15]$.

\section{CONCLUSION}

Virtual Laboratories have special requirements due to the special characteristics of online learning environment. Facilities of the laboratories in the educational institutions are generally insufficient when the huge numbers of students are considered. Implementation of a laboratory to meet the requirements has a high price. So by the use of some selected software and with minimum use of hardware, a Virtual Laboratory (VL) can be controlled remotely and can become alternatives to the traditional laboratories. In this type of laboratory, a student can have access to laboratory experiments without having any time and location limitations and can setup different parameters of the experiments from any remote locations of his or her wish. Internet connectivity is mandatory to have access to the system.

LabVIEW seems the easiest one among all the other solutions. This study may conclude that even the Vlabs supports effective and improved learning, the teacher and their interaction with students through the communication environment is an essential resource to ensure the success of the learning process in a virtual laboratory environment.

\section{REFERENCES}

[1] Zaher, M. and Dunia, R. Daly, 2008. A Unified Approach for Classroom and Laboratory Control Systems Education, 17th World Congress of the International Federation of Automatic Control, Korea, Seoul, 2008.

[2] Halvorsen, H. P. 2011. Introduction to LabVIEW, Faculty of Technology, http://home.hit.no/hansha/, 2011.

[3] Zhou1, Z. 2010. LabVIEW -Based Laboratory for Electronics Engineering Technology Program, ASEE Southeast Section Conference, pp.29-37, 2010. 
[4] Hanson, B. P., Culmer, J., and Gallagher, K. Page, E. 2009. ReLOAD: Real Laboratories Operated at a Distance, IEEE Trans on Learning Technology, Vol. 2, pp. 331-341, 2009.

[5] Hassanpour, A., Sharif, T., Ahmadian, Z. 2006. A Web Based Remote laboratory for Performing Experiments in Basic Electrical Engineering Courses, IEEE GCC Conference, pp. 1-5, March 2006.

[6] A. Mani and Patvardhan, C. 2006. A Remote Experimental Setup for Performing Experiments on Temperature Sensors, 9th International Conference on Engineering Education, July 23-28, 2006.

[7] John, A. O., Gabriel, K. G. and Patrick, K. M. 2011. Towards Virtual Laboratories: A Survey of LabVIEWBased Conduction of Science Experiments via the Internet with an Illustrative Consideration of Remote Control of an Oscilloscope, International Journal of Current Research, Vol. 3, pp. 123-127, 2011.

[8] Tiernan, P. 2010. Enhancing the learning experience of undergraduate technology students with LabVIEW software, Computer Education, Vol. 55, pp. 1579-1588, 2010.

[9] Tan, K. K., Lee, T. H. and Leu, F. M. 2000. Development of a Distant Laboratory Using LabVIEW, Int. J. Engineering Education,Vol.16, No. 3, pp. 273-282, 2000.

[10] Basher, H. A. and Isa, S. A. 2006. On-campus and Online Virtual Laboratory Experiments with LabVIEW,
IEEE Trans on Learning Technology, Vol. 41, pp. 325330, 2006.

[11] Fiesel, L. D. and Rosa, A. J. 2005. The Role of the Laboratory in Undergraduate Engineering Education, Journal of Engineering Education, Vol. 94, pp. 121-130, 2005.

[12] Davila, L., Sancristobal, E., and Castro, M., 2010. Hardware Implementation of Remote Laboratory for Digital Elctronics, IEEE Education Engineering, pp. 357362, 2010.

[13] Bhaskar, Y., Kathane, Pradeep, B. Dahikar, 2012. Development of Virtual Laboratory on Amplitude Modulation, International Journal of Computational Engineering Research, IJCER Vol. 2,Issue No.2, pp. 223-227, Mar-Apr 2012

[14] Mahanta, A. and Sarma, K. K. 2012. ICT supported Virtual Laboratory for Basic Electronics and Communication Experiments, 1st International Conference on Innovations and Advancements in Information and Communication Technology, Vol.5, pp. 219-227, 2012.

[15] Mahanta, A. and Sarma, K. K. 2012. ICT Enabled Laboratory Framework for Remote Access Applications, International Conference on Electronics and Communication Engineering, ICECE, Guwahati, India, pp. 126-130, 2012. 